\title{
SOME GENERALIZATIONS IN $H$-MODULAR SPACES OF FAN'S BEST APPROXIMATION THEOREM
}

\author{
CARLO BARDARO and RITA CEPPITELLI
}

(Received 17 May 1991; revised 15 November 1991)

Communicated by P. G. Dodds

\begin{abstract}
We state best approximation and fixed point theorems in modular spaces endowed with an $H$-space structure given by the modular topology. We consider both the cases of single valued functions and multifunctions. These theorems extend some previous results due to Ky Fan.
\end{abstract}

1991 Mathematics subject classification (Amer. Math. Soc.); primary 47 H 10; secondary 54 H 25.

\section{Introduction}

The aim of this paper is to state non-convex versions of best approximation and fixed point theorems in modular spaces endowed with its modular topology. To do that we provide the modular space with an $H$-space structure in which the linear setting is replaced by merely topological assumptions. Precisely, in this structure the convex hulls are replaced by the contractible sets. The $H$-space theory based on Horvath's ideas $[9,10]$ has been developed in $[1,2$, $3,4]$. Modular spaces with this structure are called $H$-modular spaces. This approach allows us to give a new application (Theorem 2) of our generalized KKM Theorem proved in [2] on which the main theorems of the present paper (Theorems 3, 4) are based. Moreover in Theorem 2 and so in Theorems 3 and 4 the classical compactness assumptions are relaxed as well, as in Lassonde's paper [13] and in our papers [1, 2, 3, 4]. Theorem 3 of Section 4 is a best

(C) 1994 Australian Mathematical Society 0263-6115/94 \$A2.00+0.00 
approximation theorem for single valued functions and when the modular is a seminorm in a vector space it is a non-compact and non-convex version of the Ky Fan extension [8] of Browder's fixed point theorem [5]. In Section 5 the multivalued case is considered. We introduce the concept of $H \rho$-continuity for multifunctions in terms of modular convergence and in the spirit of the Hausdorff metric and then, a relaxed convexity assumption on the modular $\rho$ compatible with the $H$-space structure (a related convexity definition can be found in [3]). These definitions, as well as other technical conditions, enable us to prove the best approximation theorem (Theorem 4 ) and, as a consequence, a fixed point property for multifunctions. Finally Theorem 4 is also compared with other generalizations to multifunctions of Ky Fan's theorem (see [15]).

\section{2. $H$-spaces}

We first recall some basic concepts in order to define the structure that we use in this paper. Further details can be found in $[9,10,1,2,3,4]$.

DEFINITIONS 1. An $H$-space is a pair $\left(X,\left\{\Gamma_{A}\right\}\right)$ where $X$ is a topological space and $\left\{\Gamma_{A}\right\}$ is a given family of non empty contractible subsets of $X$, indexed by the finite subsets of $X$, such that $A \subset B$ implies $\Gamma_{A} \subset \Gamma_{B}$. Let $\left(X,\left\{\Gamma_{A}\right\}\right)$ be an $H$-space.

A subset $D \subset X$ is called $H$-convex if for every finite subset $A \subset D$ it follows $\Gamma_{A} \subset D$. A subset $D \subset X$ is called weakly $H$-convex if, for every finite subset $A \subset D$, the intersection $\Gamma_{A} \cap D$ is nonempty and contractible.

A subset $K \subset X$ is called $H$-compact if for every finite subset $A \subset X$ there is a compact, $H$-convex set $D \subset X$ such that $K \cup A \subset D$. A subset $X_{1} \subset X$ is called compactly closed if $X_{1}$ is closed relative to every compact subset of $X$.

A multifunction $F: X \rightarrow X$ is called $H$-KKM if $\Gamma_{A} \subset \bigcup_{x \in A} F(x)$, for every finite subset $A \subset X$.

Theorem 2 below is proved as an application of the following generalization of the KKM Theorem given in [2].

THEOREM 1 [2]. Let $\left(X,\left\{\Gamma_{A}\right\}\right)$ be an $H$-space and $F: X \rightarrow X$ an $H$-KKM multifunction such that

(a) For every $x \in X, F(x)$ is compactly closed; 
(b) There is a compact set $L \subset X$ and an $H$-compact $K \subset X$ such that, for each weakly $H$-convex set $D$ with $K \subset D \subset X$, we have $\bigcap_{x \in D}(F(x) \cap D) \subset L$. Then $\bigcap_{x \in X} F(x) \neq \emptyset$.

Note that hypothesis (b) is more general than the following one:

(I) There is an $H$-compact $K \subset X$ such that $\bigcap_{x \in K} F(x)$ is compact.

Hence, if $X$ is compact, property (I) and so (b) is immediately fulfilled.

THEOREM 2. Let $\left(X,\left\{\Gamma_{A}\right\}\right)$ be an $H$-space, $D \subset X$ an $H$-convex subset. Let $G \subset D \times D$ be a subset such that

(a) For every $x \in D$, the set $\{y \in D:(x, y) \in G\}$ is compactly closed in $D$;

(b) For every $y \in D$, the set $\{x \in D:(x, y) \notin G\}$ is $H$-convex or empty;

(c) For every $x \in D,(x, x) \in G$;

(d) There is an $H$-compact $D_{0} \subset D$ such that the set $T=\{y \in D:(x, y) \in G$ for every $x \in D_{0}$ \} is compact.

Then there is $y_{0} \in T$ such that $D \times\left\{y_{0}\right\} \subset G$.

Observe that in the case of a compact space $X$, condition (d) is automatically satisfied, so the statement of Theorem 2 becomes simpler.

Proof. For every $x \in D$, let $F(x)=\{y \in D:(x, y) \in G\}$. By (a), $F(x)$ is compactly closed in $D$. By (b) and (c), if $A=\left\{x_{1}, \ldots, x_{n}\right\} \subset D$, then $\Gamma_{A} \subset \bigcup_{i=1}^{n} F\left(x_{i}\right)$. Indeed if $z \in \Gamma_{A}$ and $z \notin \bigcup_{i=1}^{n} F\left(x_{i}\right)$, then $z \notin F\left(x_{i}\right)$ for every $i=1, \ldots, n$, that is $\left(x_{i}, z\right) \notin G$, for every $i=1, \ldots, n$ and so $\Gamma_{A} \subset\{x \in D:(x, z) \notin G\}$. In particular $(z, z) \notin G$, a contradiction. Finally, by (d), $\bigcap_{x \in D_{0}} F(x)=T$ is compact. Then, by Theorem 1 there is $y_{0} \in \bigcap_{x \in D} F(x)$ that is $D \times\left\{y_{0}\right\} \subset G$.

We note that Theorem 2 was also proved in the meantime and independently by Chen [7, Theorem 1], using the same method based on our Theorem 1.

\section{Some definitions concerning modular spaces}

Let $E$ be a real vector space. A functional $\rho: E \rightarrow[0,+\infty]$ is called a modular if the following conditions are verified

(1) $\rho(x)=0$ if and only if $x=0$; 
(2) $\rho(-x)=\rho(x)$, for every $x \in E$;

(3) $\rho(\alpha x+\beta y) \leq \rho(x)+\rho(y)$, for every $\alpha, \beta \geq 0, \alpha+\beta=1$, and $x, y \in E$.

Let $E_{\rho}=\{x \in E: \rho(\lambda x)<+\infty$, for every $\lambda>0\}$. It is well known that $E_{\rho}$ is a vector subspace of $E$ (see [14]).

A sequence $\left(x_{n}\right)_{n} \subset E_{\rho}$ is said to be $\rho$-convergent or modular convergent to $x$ in $E_{\rho}$ (we denote it by $x_{n} \stackrel{\rho}{\rightarrow} x$ ) if there is $\lambda>0$ such that $\rho\left(\lambda\left(x_{n}-x\right)\right) \rightarrow 0$ for divergent $n$. A subset $A \subset E_{\rho}$ is called $\rho$-closed if for every sequence $\left(x_{n}\right)_{n} \subset A$, with $x_{n} \stackrel{\rho}{\rightarrow} x$, then $x \in A$. A subset $A \subset E_{\rho}$ is called $\rho$-compact if every sequence $\left(x_{n}\right)_{n}$ has a subsequence $\rho$-convergent in $\mathrm{A}$. The topological space $E_{\rho}$ provided with this topology will be denoted again by $E_{\rho}$.

A subset $D \subset E_{\rho}$ has the property $(\theta)$ if every sequence $\left(x_{n}\right)_{n} \rho$-convergent to $x_{0}$ in $D$ admits a subsequence $\left(x_{k_{n}}\right)_{n}$ such that $\rho\left(x_{k_{n}}\right) \rightarrow \rho\left(x_{0}\right)$ as $n$ diverges.

At first, we give an example of a subset of a modular space with property $(\theta)$. Let $(\Omega, \mathscr{F}, \mu)$ be a measurable space where $\mu$ is a non-negative, non-atomic and finite measure on $\Omega$. Let $X$ be the space of all $\mu$-measurable real functions on $\Omega$. Let us consider the Orlicz class $E^{\phi}$ with respect to the modular

$$
\rho(x)=\int_{\Omega} \phi(|x(t)|) d \mu(t), \quad x \in X,
$$

where $\phi$ is a $\phi$-function [14, page 4].

A set $D \subset E^{\phi}$ is sequentially equi-absolutely $\phi$-integrable if for every $\epsilon>0$ and every sequence $\left(x_{n}\right)_{n}$ in $D$ there is $\delta>0$, such that $\int_{A} \phi\left(\left|x_{n}(t)\right|\right) d \mu(t)<\epsilon$ for every $A \in \mathscr{F}$ with $\mu(A)<\delta$.

It is easy to show that every sequentially equi-absolutely $\phi$-integrable subset $D$ of $E^{\phi}$ has the property $(\theta)$. Indeed, if $x_{n} \stackrel{\rho}{\rightarrow} x_{0}$ in $D$, then there is a subsequence $x_{k_{n}} \rightarrow x_{0}$ almost everywhere in $\Omega$. Since $\phi$ is continuous, $\phi\left(\left|x_{k_{n}}\right|\right) \rightarrow \phi\left(\left|x_{0}\right|\right)$ almost everywhere in $\Omega$, too. By the Vitali Theorem $\rho\left(x_{k_{n}}\right) \rightarrow \rho\left(x_{0}\right)$ and so property $(\theta)$ holds.

Remark that the subspace $\rho$-topology on a subset $D \subset E_{\rho}$ with the property $(\theta)$ is weaker than the strong topology induced by the $F$-norm

$$
\|x\|_{\rho}=\inf \left\{u>0: \rho\left(\frac{x}{u}\right) \leq 1\right\} .
$$

As an example we consider the Orlicz class $E^{\phi}$ induced by $\phi(u)=e^{u}-1$, and the sequence $x_{n}:[0,1] \rightarrow \mathbb{R}$ defined by $x_{n}=n \chi_{\left[0,1 / n e^{n}\right]}$. Let $D$ be the $\rho$-closure of the set $\left\{x_{n}: n \in \mathbb{N}\right\}$. Since $x_{n} \stackrel{\rho}{\rightarrow} x_{0}=0$ in $D$, this holds for every its subsequence and so $D$ satisfies the property $(\theta)$. But $\left(\rho\left(2 x_{n}\right)\right)_{n}$ diverges. 
The aim of this paper is to state non-convex best approximation theorems in modular spaces. For this purpose we introduce in $E_{\rho}$ an $H$-space structure. For every finite subset $A \subset E_{\rho}$ let us denote by $\Gamma_{A}$ the corresponding contractible set. The space $E_{\rho}$ with this $H$-space structure will be called an $H$-modular space.

\section{A best approximation theorem for single valued functions}

In this section we state a best approximation result as well as a fixed point theorem for functions defined in $H$-convex subset of $E_{\rho}$. In order to do this, we introduce the following definition.

DEFINITION 2. Let $D$ be any subset in $E$. A function $f: D \rightarrow E_{\rho}$ is called $\rho$-continuous if for every sequence $\left(x_{n}\right)_{n} \subset D$ with $x_{n} \stackrel{\rho}{\rightarrow} x$ in $D$ we have $f\left(x_{n}\right) \stackrel{\rho}{\rightarrow} f(x)$.

Now we are ready to prove the main theorem of this section.

THEOREM 3. Let $C \subset E_{\rho}$ be an $H$-convex set and $f: C \rightarrow E_{\rho}$ a $\rho$ continuous function such that $(C-f(C))$ has the property $(\theta)$. Assume that there is an $H$ - $\rho$ compact $C_{0} \subset C$ such that the set $T=\{y \in C: \rho(x-f(y)) \geq$ $\rho(y-f(y))$, for every $\left.x \in C_{0}\right\}$ is $\rho$-compact. Finally suppose that

(+) for every $w \in E_{\rho}, \lambda \in \mathbb{R}$ the set $\left\{x \in E_{\rho}: \rho(x-w)<\lambda\right\}$ is $H$-convex.

Then there is $y_{0} \in T$ such that

$$
\rho\left(y_{0}-f\left(y_{0}\right)\right)=\min _{x \in C} \rho\left(x-f\left(y_{0}\right)\right) .
$$

ProOf. Let $G=\{(x, y) \in C \times C: \rho(x-f(y)) \geq \rho(y-f(y))\}$. By definition, condition (c) and (d) of Theorem 2 of Section 2 are fulfilled. Now, we prove that the section $G(x)=\{y \in C:(x, y) \in G\}$ is $\rho$-closed for every $x \in C$. Let $x$ be fixed in $C$.

Let $\left(y_{n}\right)_{n} \subset G(x)$ a sequence $\rho$-convergent to $y_{0}$. By $\rho$-continuity of $f$, $\left(f\left(y_{n}\right)\right)_{n} \rho$-converges to $f\left(y_{0}\right)$ and so $\left(x-f\left(y_{n}\right)\right)_{n} \rho$-converges to $\left(x-f\left(y_{0}\right)\right)$ and $\left(y_{n}-f\left(y_{n}\right)\right)_{n} \rho$-converges to $\left(y_{0}-f\left(y_{0}\right)\right)$. Since $\rho\left(x-f\left(y_{n}\right)\right) \geq$ $\rho\left(y_{n}-f\left(y_{n}\right)\right)$, by the property $(\theta)$ we have:

$$
\rho\left(x-f\left(y_{0}\right)\right) \geq \rho\left(y_{0}-f\left(y_{0}\right)\right)
$$


and so $y_{0} \in G(x)$, that is, $G(x)$ is $\rho$-closed.

Let now $y \in C$, be fixed. By property (+), the set $C \backslash G(y)=\{x \in C$ : $(x, y) \notin G\}$ is $H$-convex, if non-empty, on putting $w=f(y)$ and $\lambda=\rho(y-$ $f(y))$. Therefore by Theorem 2 there is $y_{0} \in T$ such that $C \times\left\{y_{0}\right\} \subset G$ or $\rho\left(x-f\left(y_{0}\right)\right) \geq \rho\left(y_{0}-f\left(y_{0}\right)\right)$, for every $x \in C$ and so the assertion follows.

COROLlaRY 1. (Fixed point theorem) Under the assumptions of Theorem 3, if $f(C) \subset C$, there is $y_{0} \in T$ such that $f\left(y_{0}\right)=y_{0}$.

PROOF. It is sufficient to observe that $\min _{x \in C} \rho\left(x-f\left(y_{0}\right)\right)=0$.

REMARKS. (1) In the convex case, that is, $\Gamma_{A}=\operatorname{co} A$ for every finite set $A \subset E_{\rho}$, the property (+) of Theorem 3 is equivalent to quasi-convexity of the modular $\rho$, namely the set $\left\{x \in E_{\rho}: \rho(x)<\lambda\right\}$ is convex for every $\lambda \in \mathbb{R}$.

(2) If the $H$-space structure in $E_{\rho}$ is translation invariant, that is, for every finite subset $A \subset E_{\rho}$ and $y \in E_{\rho}$, we have $\Gamma_{A}-y=\Gamma_{A-y}$, then the property (+) is equivalent to saying that the set $\left\{x \in E_{\rho}: \rho(x)<\lambda\right\}$ is $H$-convex, for every $\lambda \in \mathbb{R}$.

(3) When $\rho$ is an $F$-norm in a vector space $E$, with $\rho(\alpha x) \leq \rho(x)$ for every $x \in E, \alpha \in[0,1]$, every subset $D \subset E$ has the property $(\theta)$ with respect to strong convergence. Thus, in this particular case, Theorem 3 is a non-compact and non-convex version for the homogeneous subadditive functionals of the $\mathrm{Ky}$ Fan Extension of Browder's fixed point theorem [8].

(4) In Theorem 3 the property $(\theta)$ assumed on $C-f(C)$ can be relaxed to the following one:

for all sequences $\left(x_{n}\right)_{n},\left(y_{n}\right)_{n}$ from $C-f(C) \rho$-convergent to $x_{0}$, $y_{0}$ respectively with $\rho\left(x_{n}\right) \geq \rho\left(y_{n}\right)$, we have $\rho\left(x_{0}\right) \geq \rho\left(y_{0}\right)$.

It is easy to show that the property $(\theta)$ implies the property $\left(\theta^{*}\right)$.

(5) All the results of Section 4 can be extended to the whole modular space $L_{\rho}$, namely $L_{\rho}=\{x \in E: \rho(\lambda x) \rightarrow 0\}$. In this case it is sufficient to require in Theorem 3 the following further condition: for every $y \in C$, there is $x \in C$ such that $\rho(x-f(y))<+\infty$.

\section{A best approximation theorem for multifunctions}

In this section we state a best approximation result as well as a fixed point theorem for multifunctions defined in $H$-convex subsets of $E_{\rho}$. In order to do 
this, we will introduce some background concepts.

Given a subset $K \subset E_{\rho}$ we set $\rho(K, x)=\inf _{t \in K} \rho(t-x)$.

Let $2^{E_{\rho}}$ be the family of non-empty subsets of $E_{\rho}$ and for $A, B \in 2^{E_{\rho}}$,

$$
e_{\lambda}(A, B)=\operatorname{supinf}_{a \in A} \rho(\lambda(a-b))
$$

DEFINITION 3. A sequence $\left(A_{n}\right)_{n=0,1,2, \ldots,} A_{n} \in 2^{E_{\rho}}$, is said to be $\rho$-convergent to $A_{0}$ in the sense of Hausdorff (H $\rho$-convergent) if there is $\lambda>0$ such that $\lim _{n \rightarrow+\infty} \max \left(e_{\lambda}\left(A_{n}, A_{0}\right), e_{\lambda}\left(A_{0}, A_{n}\right)\right)=0$. We will write briefly $A_{n} \stackrel{H_{\rho}}{\rightarrow} A_{0}$.

DEFINITION 4. A multifunction $L: E_{\rho} \rightarrow 2^{E_{\rho}}$ is said to be continuous in the sense of Hausdorff (Ho-continuous) if $L\left(y_{n}\right) \stackrel{H \rho}{\rightarrow} L\left(y_{0}\right)$ whenever $y_{n} \stackrel{\rho}{\rightarrow} y_{0}$.

The following result gives some properties of $H \rho$-continuous multifunctions.

PROPOSITION 1. Let $L: E_{\rho} \rightarrow 2^{E_{\rho}}$ be an H $\rho$-continuous multifunction with $\rho$-closed values. If $y_{n} \stackrel{\rho}{\rightarrow} y_{0}$ in $E_{\rho}$, then the following properties hold:

(i) for every $z_{0} \in L\left(y_{0}\right)$ there is $z_{n} \in L\left(y_{n}\right)$ such that $z_{n} \stackrel{\rho}{\rightarrow} z_{0}$;

(ii) for every sequence $\left(z_{n}\right)_{n=0,1,2, \ldots}$ with $z_{n} \in L\left(y_{n}\right)$ for $n \geq 1$ and $z_{n} \stackrel{\rho}{\rightarrow} z_{0}$ then $z_{0} \in L\left(y_{0}\right)$.

PROOF. (i) Let $z_{0} \in L\left(y_{0}\right)$ be fixed. By $H \rho$-continuity of $L$, from $y_{n} \stackrel{\rho}{\rightarrow} y_{0}$ it follows that $L\left(y_{n}\right) \stackrel{H \rho}{\rightarrow} L\left(y_{0}\right)$; thus there is $\lambda>0$ such that $\lim _{n \rightarrow+\infty} e_{\lambda}\left(L\left(y_{0}\right)\right.$, $\left.L\left(y_{n}\right)\right)=0$. So, for every $\epsilon>0$ there is $\bar{n}_{\epsilon}$ such that $\sup _{z \in L\left(y_{0}\right)} \inf _{t \in L\left(y_{n}\right)}$ $\rho(\lambda(z-t))<\epsilon$, for every $n>\bar{n}_{\epsilon}$, from which inf $\operatorname{tiL}_{t\left(y_{n}\right)} \rho\left(\lambda\left(z_{0}-t\right)\right)<\epsilon$, for every $n>\bar{n}_{\epsilon}$. Hence for every $n>\bar{n}_{\epsilon}$ there is $t_{n} \in L\left(y_{n}\right)$ such that $\rho\left(\lambda\left(z_{0}-t_{n}\right)\right)<\epsilon$ that is $t_{n} \stackrel{\rho}{\rightarrow} z_{0}$.

(ii) Let $\left(z_{n}\right)_{n=0,1,2, \ldots}$ with $z_{n} \in L\left(y_{n}\right), n \geq 1$ and $z_{n} \stackrel{\rho}{\rightarrow} z_{0}$. By $H \rho$-continuity of $L$ there is $\lambda>0$ such that $\lim _{n \rightarrow+\infty} e_{\lambda}\left(L\left(y_{n}\right), L\left(y_{0}\right)\right)=0$. So for every $\epsilon>0$ there is $\bar{n}_{\epsilon}$ such that for every $n>\bar{n}_{\epsilon}$ and $t \in L\left(y_{n}\right)$ we have $\inf _{z \in L\left(y_{0}\right)} \rho(\lambda(t-z))<\epsilon$. In particular $\inf _{z \in L\left(y_{0}\right)} \rho\left(\lambda\left(z_{n}-z\right)\right)<\epsilon$ where $z_{n} \epsilon$ $L\left(y_{n}\right)$ and $z_{n} \stackrel{\rho}{\rightarrow} z_{0}$. Hence for every $z_{n} \in L\left(y_{n}\right), n>\bar{n}_{\epsilon}$, there is $\bar{z}_{n} \in L\left(y_{0}\right)$ such that $\rho\left(\lambda\left(z_{n}-\bar{z}_{n}\right)\right)<\epsilon / 2$. From $z_{n} \stackrel{\rho}{\rightarrow} z_{0}$ it follows $\lim _{n \rightarrow+\infty} \rho\left(\tilde{\lambda}\left(z_{n}-z_{0}\right)\right)=0$ for some $\tilde{\lambda}>0$ and so we can assume $\rho\left(\tilde{\lambda}\left(z_{n}-z_{0}\right)\right)<\epsilon / 2$, for every $n>\bar{n}_{\epsilon}$. Putting $\mu=\min \{\lambda, \tilde{\lambda}\}$, by properties of $\rho$ we have for $n>\bar{n}_{\epsilon}$,

$$
\rho\left(\frac{\mu}{2}\left(\bar{z}_{n}-z_{0}\right)\right)=\rho\left(\frac{\mu}{2}\left(\bar{z}_{n}-z_{n}+z_{n}-z_{0}\right)\right) \leq \rho\left(\mu\left(\bar{z}_{n}-z_{n}\right)\right)+\rho\left(\mu\left(z_{n}-z_{0}\right)\right)<\epsilon
$$


and so $\bar{z}_{n} \stackrel{\rho}{\rightarrow} z_{0}$. The assertion follows by $\rho$-closedness of $L\left(y_{0}\right)$.

Now, we introduce the following generalization of the convexity of the modular $\rho$. Let

$$
\Sigma^{(n-1)}=\left\{\left(t_{1}, \ldots, t_{n}\right) \in \mathbb{R}^{n}: \sum_{i=1}^{n} t_{i}=1 ; t_{i} \geq 0, i=1, \ldots, n\right\}
$$

be the $(n-1)$-dimensional standard simplex. We say that $\rho$ has the property $(+)$ if given $A=\left\{x_{1}, \ldots, x_{n}\right\} \subset E_{\rho}, B=\left\{y_{1}, \ldots, y_{n}\right\} \subset E_{\rho}$, for every $z \in \Gamma_{A}$ there are $\xi \in \Gamma_{B}$ and $\left(t_{1}(z, \xi), \ldots, t_{n}(z, \xi)\right) \in \Sigma^{(n-1)}$ such that $\rho(z-\xi) \leq$ $\sum_{i=1}^{n} t_{i}(z, \xi) \rho\left(x_{i}-y_{i}\right)$.

Note that in the convex case, that is, $\Gamma_{A}=\operatorname{co} A$ for every finite subset $A \subset E_{\rho}$, the property $(+)$ generalizes the convexity of $\rho$.

In [3] we introduced the following concept of $H$-convexity: for every $\Delta=$ $\left\{w_{1}, \ldots, w_{n}\right\} \subset E_{\rho}$, and for every $\eta \in \Gamma_{\Delta}$ there is an $n$-tuple $\left(t_{1}(\eta), \ldots, t_{n}(\eta)\right) \in$ $\Sigma^{(n-1)}$ such that $\rho(\eta) \leq \sum_{i=1}^{n} t_{i}(\eta) \rho\left(w_{i}\right)$.

In order to compare the property $(+)$ with $H$-convexity we remark the following.

Given $A=\left\{x_{1}, \ldots, x_{n}\right\}, B=\left\{y_{1}, \ldots, y_{n}\right\}$, let $\Delta=\left\{x_{1}-y_{1}, \ldots, x_{n}-y_{n}\right\}$. If for every $z \in \Gamma_{A}$ there is $\xi \in \Gamma_{B}$ such that $z-\xi \in \Gamma_{\Delta}$, then $H$-convexity implies the property $(+)$.

However the following proposition holds.

PROPOSITION 2. If $\rho$ satisfies the property $(+)$, then the function $\rho(K, \cdot)$ is $H$-convex in $E_{\rho}$ for every $H$-convex $K \subset E_{\rho}$.

PRoof. Let $A=\left\{x_{1}, \ldots, x_{n}\right\} \subset E_{\rho}$ be fixed. For every $m \in \mathbb{N}^{+}$there is $T_{m}=\left\{\tau_{1}^{m}, \ldots, \tau_{n}^{m}\right\} \subset K$ such that

$$
\rho\left(K, x_{i}\right)>\rho\left(\tau_{i}^{m}-x_{i}\right)-\frac{1}{m}, \quad i=1, \ldots, n .
$$

For a fixed $\xi \in \Gamma_{A}$ and $m \in \mathbb{N}^{+}$, by property (+) there is $\tau_{m}^{*} \in \Gamma_{T_{m}} \subset K$ and $\left\{t_{1}\left(\tau_{m}^{*}, \xi\right), \ldots, t_{n}\left(\tau_{m}^{*}, \xi\right)\right\}$ in $\Sigma^{n-1}$ such that

$$
\rho(K, \xi) \leq \rho\left(\tau_{m}^{*}-\xi\right) \leq \sum_{i=1}^{n} t_{i}\left(\tau_{m}^{*}, \xi\right) \rho\left(\tau_{i}^{m}-x_{i}\right) .
$$

For every $m \in \mathbb{N}^{+}$we put $t^{m}=\left(t_{1}\left(\tau_{m}^{*}, \xi\right), \ldots, t_{n}\left(\tau_{m}^{*}, \xi\right)\right)$. By the compactness of $\Sigma^{n-1}$, the sequence $\left(t^{m}\right)_{m \in \mathbb{N}^{+}}$admits a subsequence still denoted 
by $\left(t^{m}\right)_{m \in \mathbb{N}}$ convergent to $\bar{t}=\left(\bar{t}_{1}(\xi), \ldots, \bar{t}_{n}(\xi)\right) \in \Sigma^{n-1}$. By (1) and (2) $\rho(K, \xi)<\sum_{i=1}^{n} t_{i}\left(\tau_{m}^{*}, \xi\right) \rho\left(K, x_{i}\right)+1 / m$ for every $m \in \mathbb{N}^{+}$and so $\rho(K, \xi) \leq$ $\sum_{i=1}^{n} \bar{t}_{i}(\xi) \rho\left(K, x_{i}\right)$, as asserted.

Finally we will use the following elementary result.

PROPOSITION 3. Let $X$ be any topological space, $\phi: X \rightarrow \mathbb{R}^{+}$be a given function such that for every sequence $\left(x_{n}\right)_{n}$ convergent to $x_{0}$ in $X$ there is a subsequence $\left(x_{k_{n}}\right)_{n}$ such that $\phi\left(x_{k_{n}}\right) \rightarrow \phi\left(x_{0}\right)$. Then, if $X$ is compact, $\phi$ attains its minimum value.

Let $C \subset E_{\rho}$ be an $H$-convex set and $L$ be a multifunction defined on $C$. From now on we suppose that

(i) the set $L(C)=\bigcup_{x \in C} L(x)$ is $\rho$-compact;

(ii) the set $C-L(C)$ has the property $(\theta)$;

(iii) the multifunction $L$ is $H \rho$-continuous with non-empty $\rho$-closed and $H$-convex values.

We refer to these assumptions as hypotheses (I).

The following lemma gives a key result for the main theorem of this section (Theorem 4).

LEMMA 1. Suppose hypotheses (I) hold. If $\left(y_{n}\right)_{n} \subset C$ and $y_{n} \stackrel{\rho}{\rightarrow} y_{0}$ in $C$; then there is a subsequence $\left(y_{k_{n}}\right)_{n}$ such that $\rho\left(L\left(y_{k_{n}}\right), x\right) \rightarrow \rho\left(L\left(y_{0}\right), x\right)$ for every $x \in C$.

PROOF. For a fixed $x \in C$, and $n \in \mathbb{N}$ we define $\phi(t)=\rho(x-t), t \in L\left(y_{n}\right)$. The function $\phi$ satisfies the hypotheses of Proposition 3. Indeed, let $\left(t_{k}\right)_{k} \subset$ $L\left(y_{n}\right)$ be a sequence $\rho$-convergent to $t_{0} \in L\left(y_{n}\right)$. As $\left(x-t_{k}\right) \stackrel{\rho}{\rightarrow}\left(x-t_{0}\right)$ in $C-L\left(y_{n}\right)$, then by (ii) there is a subsequence $\left(t_{h_{k}}\right)_{k}$ such that $\rho\left(x-t_{h_{k}}\right) \rightarrow$ $\rho\left(x-t_{0}\right)$, that is, $\phi\left(t_{h_{k}}\right) \rightarrow \phi\left(t_{0}\right)$. So by Proposition 3 and the $\rho$-compactness of $L\left(y_{n}\right)$ there is $\xi_{n} \in L\left(y_{n}\right)$ such that inf $t_{t \in L\left(y_{n}\right)} \rho(x-t)=\rho\left(x-\xi_{n}\right)$.

Let us consider the sequence of minimizing elements $\left(\xi_{n}\right)_{n}$. By $\rho$-compactness of $L(C)$ there is a subsequence $\left(\xi_{k_{n}}\right)_{n}$ and $\xi_{0} \in L(C)$ such that $\xi_{k_{n}} \stackrel{\rho}{\rightarrow} \xi_{0}$ and $\rho\left(\xi_{k_{n}}-x\right) \rightarrow \rho\left(\xi_{0}-x\right)$. By $H \rho$-continuity of $L, \xi_{0} \in L\left(y_{0}\right)$. So we have

$$
\lim _{n \rightarrow+\infty} \rho\left(L\left(y_{k_{n}}\right), x\right)=\rho\left(\xi_{0}-x\right) \geq \rho\left(L\left(y_{0}\right), x\right) .
$$

Next, we prove that equality occurs. If it is false, there is a $\delta>0$ and $\bar{n} \in \mathbb{N}$ such that for every $n>\bar{n}$ we have $\rho\left(L\left(y_{k_{n}}\right), x\right)-\rho\left(L\left(y_{0}\right), x\right)>\delta$ and so

$$
\rho(t-x)-\rho\left(L\left(y_{0}\right), x\right)>\delta, \quad \text { for every } t \in L\left(y_{k_{n}}\right) .
$$


Let $z_{0} \in L\left(y_{0}\right)$ be fixed. By $H \rho$-continuity of $L$ there is a sequence $\left(z_{k_{n}}\right)_{n}$ with $z_{k_{n}} \in L\left(y_{k_{n}}\right)$ such that $z_{k_{n}} \stackrel{\rho}{\rightarrow} z_{0}$ and by (3)

$$
\rho\left(z_{k_{n}}-x\right)-\rho\left(L\left(y_{0}\right), x\right)>\delta, \quad \text { for every } n \in \mathbb{N} \text {. }
$$

Finally, by (ii) using a further subsequence, we have $\rho\left(z_{0}-x\right)-\rho\left(L\left(y_{0}\right), x\right) \geq \delta$, for every $z_{0} \in L\left(y_{0}\right)$, a contradiction.

THEOREM 4. Suppose that property (+) on $\rho$ and hypotheses (I) hold. If there is an $H$ - $\rho$ compact $C_{0} \subset C$ such that the set $T=\{y \in C$ : there is $z \in$ $L(y)$ with $\rho(L(y), x) \geq \rho(z-y)$, for every $\left.x \in C_{0}\right\}$ is $\rho$-compact, then there is $y_{0} \in T$ such that

$$
\min _{z \in L\left(y_{0}\right)} \rho\left(y_{0}-z\right)=\inf _{x \in C} \rho\left(L\left(y_{0}\right), x\right)
$$

Observe that if $C$ is $\rho$-compact the existence of such a subset $C_{0}$ is automatically satisfied, so the statement of Theorem 4 becomes simpler.

Proof. Let $G=\{(x, y) \in C \times C$ : there is $z \in L(y)$ with $\rho(L(y), x) \geq$ $\rho(z-y)\}$. At first, we prove that $(x, x) \in G$, for every $x \in C$. Fixed $x \in C$, let $\phi: L(x) \rightarrow \mathbb{R}^{+}$defined by $\phi(t)=\rho(x-t)$. As proved in Lemma 1 , the function $\phi$ satisfies the hypotheses of Proposition 3. So, there is $z \in L(x)$ such that $\rho(x-z)=\min _{t \in L(x)} \rho(x-t) \equiv \rho(L(x), x)$, that is, $(x, x) \in G$.

Next, we prove that for every fixed $x \in C$ the set $G(x)=\{y \in C$ : there is $z \in L(y)$ with $\rho(L(y), x) \geq \rho(z-y)\}$ is $\rho$-closed.

Let $\left(y_{n}\right)_{n} \subset G(x)$ with $y_{n} \stackrel{\rho}{\rightarrow} y_{0}$. For every $n \in N$, let $z_{n} \in L\left(y_{n}\right)$ such that

$$
\rho\left(L\left(y_{n}\right), x\right) \geq \rho\left(z_{n}-y_{n}\right)
$$

By (i) and (iii) there is a subsequence $\left(z_{k_{n}}\right)_{n} \rho$-convergent to $z_{0} \in L\left(y_{0}\right)$. By (ii) and Lemma 1 we can suppose, up to subsequences, that $\rho\left(z_{k_{n}}-y_{k_{n}}\right) \rightarrow$ $\rho\left(z_{0}-y_{0}\right)$ and $\rho\left(L\left(y_{k_{n}}\right), x\right) \rightarrow \rho\left(L\left(y_{0}\right), x\right)$. Hence by (5)

$$
\rho\left(L\left(y_{0}\right), x\right)=\lim _{n} \rho\left(L\left(y_{k_{n}}\right), x\right) \geq \lim _{n} \rho\left(z_{k_{n}}-y_{k_{n}}\right)=\rho\left(z_{0}-y_{0}\right),
$$

that is, $G(x)$ is $\rho$-closed.

Finally, we prove that for every $y \in C$ the set

$$
C \backslash G(y)=\{x \in C: \text { for every } z \in L(y), \rho(L(y), x)<\rho(z-y)\}
$$


is $H$-convex.

Let $y \in C$ and $z \in L(y)$ be fixed. Let $A=\left\{x_{1}, \ldots, x_{n}\right\} \subset C \backslash G(y)$, then

$$
\rho\left(L(y), x_{i}\right)<\rho(z-y), \quad \text { for every } i=1, \ldots, n \text {. }
$$

For a fixed $\xi \in \Gamma_{A}$, by Proposition 2, $H$-convexity of $L(y)$ and (6), there is $\left(\bar{t}_{1}(\xi), \ldots, \bar{t}_{n}(\xi)\right) \in \Sigma^{n-1}$ such that

$$
\rho(L(y), \xi) \leq \sum_{i=1}^{n} \bar{t}_{i}(\xi) \rho\left(L(y), x_{i}\right)<\rho(z-y),
$$

which is the $H$-convexity of $C \backslash G(y)$.

Finally, hypothesis (d) of Theorem 1 is automatically fulfilled. By Theorem 1, the assertion follows.

COROLlaRY 2. (Fixed point theorem) Under the assumptions of Theorem 4, if $L(C) \subset C$, there is $y_{0} \in T$, such that $y_{0} \in L\left(y_{0}\right)$.

PROOF. Let $y_{0} \in T$ satisfy (4). If $x \in L\left(y_{0}\right) \subset C$ then

$$
\inf _{z \in L\left(y_{0}\right)} \rho(x-z)=0, \quad \text { and so } \quad \inf _{x \in C} \inf _{z \in L\left(y_{0}\right)} \rho(x-z)=0 .
$$

Therefore the assertion follows again by (4).

REMARKS. Theorem 4 is an extension in various directions (non-convex and non-compact case, multifunctions, non-homogeneous functionals) of Ky Fan's extension of Browder's fixed point theorem [8].

Among the other generalizations to multifunctions of the Ky Fan theorem [8], we limit ourselves to quote Theorem 1 of Sehgal-Singh in [15]. There, the authors work with convex subsets in a topological vector space and use a relaxed compactness hypothesis, different with respect to our one.

In the compact case, our theorem is an extension to $H$-modular spaces of the Sehgal-Singh theorem.

Finally we note that all the results of Section 5 can be extended to the whole modular space $L_{\rho}$, namely, $L_{\rho}=\left\{x \in E: \lim _{\lambda \rightarrow 0} \rho(\lambda x)=0\right\}$. In this case it is sufficient to require in Theorem 4 the following further condition: for every $x, y \in C$, there is $z \in L(y)$ such that $\rho(z-x)<+\infty$. 


\section{References}

[1] C. Bardaro and R. Ceppitelli, 'Minimax inequalities in Riesz spaces', Atti. Sem. Mat. Fis. Univ. Modena 35 (1987), 63-70.

[2] — - 'Some further generalizations of Knaster-Kuratowski-Mazurkiewicz Theorem and minimax inequalities', J. Math. Anal. Appl. 132 (1988), 484-490.

[3] — 'Applications of the generalized Knaster-Kuratowski-Mazurkiewicz Theorem to variational inequalities', J. Math. Anal. Appl. 137 (1989), 46-58.

[4] , 'Fixed point theorems and vector valued minimax theorems', J. Math. Anal. Appl. 146 (1990), 363-373.

[5] F. E. Browder, 'A new generalization of the Schauder fixed point theorem', Math. Ann. 174 (1967), 285-290.

[6] C. Castaing and M. Valadier, Convex analysis and measurable multifunctions, Lecture Notes in Math. 580 (Springer, Berlin, 1977).

[7] G. Y. Chen, 'Generalized section theorem and minimax inequality for a vector-valued mapping', to appear.

[8] K. Fan, 'Extensions of two fixed point theorems of F. E. Browder', Math. Z. 112 (1969), 234-240.

[9] C. D. Horvath, 'Points fixes et coincidences dans les éspaces topologiques compacts contractiles', C. R. Acad. Sci. Paris Sér. I Math. 299 (1984), 519-521.

[10] - Some results on multivalued mappings and inequalities without convexity, Lecture Notes in Pure and Appl. Math. 107 (Marcel Dekker, New York, 1988).

[11] _ ' 'Contractibility and generalized convexity', J. Math. Anal. Appl. 156 (1991), 341357.

[12] W. M. Kozlowski, Modular function spaces (Marcel Dekker, New York, 1989).

[13] M. Lassonde, 'On the use of K.K.M. multifunctions in fixed point theory and related topics', J. Math. Anal. Appl. 97 (1983), 151-201.

[14] J. Musielak, Orlicz spaces and modular spaces, Lecture Notes in Math. 1034 (Springer, Berlin, 1983).

[15] V. M. Sehgal and S. P. Singh, 'A generalization to multifunctions of Fan's best approximation theorem', Proc. Amer. Math. Soc. 102 (1988), 534-537.

Dipartimento di Matematica

Università degli Studi di Perugia

via Pascoli

06100 Perugia

Italy
Dipartimento di Matematica e Fisica

Università di Camerino via Venanzi 16 62032 Camerino (MC) Italy 